\title{
Chloroquine Related to Malaria and its Promising Applications for Covid-19 Treatment
}

Luciano Barreto Silva1', Alberto Nicodemus Gomes Lopes, ${ }^{2,3, *}$

\section{ORCID IDs}

Lopes ANG (D) https://orcid.org/0000-0002-1437-9619

\begin{abstract}
Objective: this article described the use of chloroquine as an antimalarial agent with potential antiviral indications for COVID-19 infections Methods: this article consisted of online searches and gray literature whose database included PUBMED Central, BVS/BIREME, Web of Science, Science Direct, Higher Level Personnel Improvement Coordinator (CAPES), Periodic Door (Portal de Periódicos da CAPES, The Cochrane Library, and PROSPERO). Results: chloroquine and hydroxychloroquine have shown appropriate clinical reports when associated with the antibiotic Azithromycin. It has been authorized for the clinical treatment of severe acute forms of COVID infections by countries such as Brazil and the USA. Conclusions: Chloroquine seems to have potential antiviral properties that may be useful in the treatment of these severe acute forms of COVID-19 associated with Azithromycin. Nevertheless, its indication must include ECG monitoring due to the risk of prolongation of QT interval, leading to sudden cardiac death.
\end{abstract}

KEYWORDS: Cloroquine; COVID-19; QT interval. 


\section{INTRODUCTION}

The first reports of the plant that originated chloroquine appeared in Peru, used by Indian tribes who used the bark of the trees of the chincona officinalis plant to make tea, to fight chills and fever in the 17 th century ${ }^{1}$. The first reports of this plant appeared in 1633, basically indicated for the treatment against malaria. Today, more than 200 million people suffer from malaria, and more than 1 million deaths per year are in fact caused by malaria (LEMKE et al., 1995). The first scientific reports appeared in the years of the rise of Nazism just before World War II. Chloroquine was officially "discovered" in 1934 by Hans Andersaq under the name "Resochin", developed by Beyer laboratories³.

In 2019, COVID-19, an infectious disease caused by severe acute respiratory syndrome coronavirus 2 spread all over the world (Sars-CoV-2). This paper was conceived in the midst of the pandemic. The disease was first identified in Wuhan, Hubei Province, People's Republic of China, on December $1^{\text {st }}, 2019$, and the first case was reported on December $31^{\text {st }}$ of the same year. The virus is believed to be of zoonotic origin, as the first confirmed cases were mainly related to the Huanan Seafood Wholesale Market, where live animals are sold.

The aim of this work is to relate chloroquine in the treatment of infections caused by COVID-19 through a literature review.

\section{Chloroquine and its congeners}

Chloroquine is part of a large series of 4-amino-quinolines that have been thoroughly studied in the vast antimalarial research program conducted in the countryside of the United States in the middle of World War II ${ }^{4}$. The aim of the American program was to discover and use suppressant drugs that were more effective and less toxic than quinacrine, an acridine derivative that is no longer used in malaria chemotherapy due to its toxicity and the inability to cure malaria caused by Plasmaodium vivax, or acting as a causal prophylactic ${ }^{5}$. Although 4-aminoquinolins had been previously described as potential antimalarials by Russian researchers, no greater attention was paid to this chemical group, until the French reported that 3-methyl-7-chlorine-4 (4-diethylamino-1-methylbutylamino) quinoline (SN-6911; sontochin, sontoquin) was well tolerated and had great activity against human malaria ${ }^{6}$. Thus, in 1943, thousands of these compounds were synthesized and tested for malaria activity in birds and toxicity in mammals. From this series, ten of them were then studied in human volunteers with experimentally induced malaria. Of these, chloroquine has shown to be the most promising drug and has been released for clinical trials.

\section{Antiviral potential of chloroquine}

As of the time of writing this article, no specific therapy has been indicated for Sars-CoV-2, the virus responsible for COVID-19. There are a number of agents under clinical trials, but the fact is that the efficacy of any drug has not yet been established. Nevertheless, chloroquine, in the form of hydroxychloroquine, has shown promising results when administered to patients under intensive care therapy. The reason for the rediscovery of chloroquine reemerged during the SARS epidemic caused by SARS coronavirus 1, the previous member of the vast Coronoviridae family. Due to the rise of the pandemic and the thousands of dead patients, especially in Italy, some countries, such as Brazil and the USA, have authorized its prescription in clinical trials. The fact is that some drugs are being tested, alone or in combination with other drugs. The main ones are ${ }^{7}$ :

a) Chloroquine - Limited in vitro clinical data and trials suggest a potential benefit when used alone;

b) Hydroxychloroquine - Limited in vitro clinical data carried out with a low number of patients suggest potential benefit; and

c) Azithromycin - Has been associated with hydroxychloroquine and is now being tested in COVID-19 therapy with positive results. Its use as an adjuvant therapy has brought hope for the treatment of the patients.

Chloroquine and hydroxychloroquine have shown antiviral effects. Their mechanism of action is based on increased $\mathrm{pH}$ of lysosomes and cellular endorsements, resulting in the impairment of virus release from within them. Since the vast majority of viruses require low $\mathrm{pH}$ to be released into the cellular cytoplasm, all clinical evidence suggest that this 
is the mechanism by which chloroquine may play this important role in the therapy ${ }^{8}$. Thus, the virus is unable to release its genetic material inside the host cell and replicate ${ }^{9,10}$. The association with azithromycin has been indicated for the capacity of this antibiotic to treat many different types of infections caused by bacteria, such as respiratory, skin, ear, eye, and sexually transmitted infections.

\section{Chloroquine and QT interval of the electrocardiogram}

The normal heart beats regularly and well-coordinated, as the electrical impulses generated and transmitted by myocytes with unique electrical properties trigger a sequence of organized myocardial contractions, sufficient for adequate blood pumping. Chloroquine has been reported as able to prolong QT intervals in electrocardiograms (ECG).

The long QT interval, which may be congenital or drug-induced, leads to malignant ventricular arrhythmia, known as torsades de pointes.

Long QT predisposes to arrhythmias due to prolonged myocyte repolarization, a fact that induces early postdepolarization and refractory spatial dispersion. Arrhythmias and conduction changes are caused by abnormalities in the generation or conduction of these electrical impulses, or both. It is important to highlight that the increase in the QT interval is in fact able to cause fatal arrhythmia in $10 \%$ of the patients ${ }^{11}$. Therefore, it is important, if not essential, that ECG monitoring be systematically incorporated in case these drugs are implemented in the conventional treatment. The risk exists and, since coronavirus mortality rate is around 3\% in most countries, the treatment offered must never be worse than the illness itself. The panic caused by the rise of the pandemic does not justify hasty medical conducts taken based on weak evidence, as did some studies published with a low number of patients.

Another research studied 46 rheumatic patients (42 using chloroquine and 4 using hydroxychloroquine); in both groups the dosage was $7 \mathrm{mg} / \mathrm{kg} / \mathrm{day}$ of hydroxychloroquine and $4 \mathrm{mg} / \mathrm{kg} /$ day of chloroquine). Their results showed that prolongation of the QTc interval was observed in $17.39 \%$ of patients. All patients with abnormal results, except 1 , repeated the ECG with return to normal values. The authors concluded that patients using chloroquine may indeed have prolongation of the QT interval as an adverse effect of the drug ${ }^{12}$.

One of the most dangerous things concerning the use of chloroquine is undoubtedly the fact that excessive QT prolonged interval implies an inherent risk of sudden cardiac death (SCD). The dispersion of repolarization leads to oscillation on the membrane potential immediately after depolarization, resulting in electrical instability and development of the polymorphic ventricular tachycardia, Torsades de Points, leading to $\mathrm{SCD}^{13}$.

\section{CONCLUSIONS}

Chloroquine seems to have potential antiviral properties that may be useful in the treatment of the severe acute forms of COVID-19 infections, when used in association with Azithromycin. Its indication, however, must be done carefully, with adequate monitoring of the heart rhythm, due to the risk of cardiac QT prolongation able to cause sudden deaths. Periodic ECG must be included in the protocols for patients on prescription of this drug.

\section{REFERENCES}

1. Fern K. Cinchona officinalis - L. Plans for a Future. 2010-2020.

2. Lemke, T L, Williams, D A, Foye, W O Principles of medicinal chemistry. Williams \& Wilkins, 1995.

3. Krafts K, Hempelmann E, Skórska-Stania A. From methylene blue to chloroquine: a brief review of the development of an antimalarial therapy. Parasitol Res. 2012;111(1):1-6. https://doi.org/10.1007/s00436-012-2886-x 
4. Wallace DJ. Antimalarial agents and lupus. Rheum Dis Clin North Am. 1994;20(1): 243-63.

5. Rynes Rl. Antimalarial drugs in the treatment of rheumatological diseases. Br J Rheumatol. 1997;36(7):799-805. https://doi. org/10.1093/rheumatology/36.7.799

6. Peters W. Chemotherapy and drug resistance in malaria. Ipswich, Academic Press, 1970. 876 p.

7. Smith T, Bushek J, Prosser T. COVID-19 Drug therapy - Potential Options. Elsevier, 2020.

8. Savarino A, Boelaert JR, Cassone A, Majori G, Cauda R. Effects of chloroquine on viral infections: an old drug against today's diseases. The Lancet. Infectious Diseases. 2003;3(11):722-7. https://doi.org/10.1016/S1473-3099(03)00806-5

9. Al-Bari MA. Targeting endosomal acidification by chloroquine analogs as a promising strategy for the treatment of emerging viral diseases. Pharmacol Res Perspect. 2017;5(1):e00293. https://doi.org/10.1002/prp2.293

10. Fredericksen BL, Wei BL, Yao J, Luo T, Garcia JV. Inhibition of endosomal/lysosomal degradation increases the infectivity of human immunodeficiency virus. J Virol. 2002;76(22):11440-6. https://doi.org/10.1128/JVI.76.22.11440-11446.2002

11. Haeusler IL, Chan XHS, Guérin PJ, White NJ. The arrhythmogenic cardiotoxicity of the quinoline and structurally related antimalarial drugs: A systematic review. BMC Med. 2018;16(1):200. https://doi.org/10.1186/s12916-018-1188-2

12. Rey LD, Anderson B, Leonardo G, Silva MB, Skare TL, Silva JA. ECT QT interval prolongation in rheumatic patients using antimalarial drugs. Rev Bras Reumatol. 2003;43(5):275-8. https://doi.org/10.1590/S0482-5004200300500003

13. January C, Riddle JM. Early afterdepolarizations: mechanism of induction and block. A role for L-type Ca2+ current. Circ Res. 1989;64(5):977-90. https://doi.org/10.1161/01.res.64.5.977 